Murakami J.

Osaka J. Math.

24 (1987), 745-758

\title{
THE KAUFFMAN POLYNOMIAL OF LINKS AND REPRESENTATION THEORY*
}

\author{
JUN MURAKAMI
}

(Received July 3, 1986)

1. Introduction. In 1984, V. Jones [8] introduced a new polynomial invariant of link isotopy types which is now called the (one variable) Jones polynomial, which was subsequently generalized to the two variable Jones polynomial [4], [13]. These invariants are closely related to the traces of irreducible representations of Iwahori algebras (or Hecke algebras) [2], [7] associated with the symmetric groups (see [5], [9]).

The purpose of this paper is to show that the Kauffman polynomial [12] can also be interpreted as a function $F$ on a certain associative algebra. We define a knit semi-group $D_{n}$ of degree $n$ which is generated by the generators of the braid group $B_{n}$ on $n$ strings and elements $e_{1}, e_{2}, \cdots, e_{n-1}$ in Figure 4 . We call an element of $D_{n}$ an $n$-knit. We get a link $d^{\wedge}$ in the 3 -sphere by closing an $n$-knit $d$. We call $d^{\wedge}$ a closed $n$-knit coming from $d$. We also define an algebra $E_{n}(\alpha, \beta)$ for non-zero complex numbers $\alpha, \beta \in C-\{0\}$ as a quotient of a semi-group algebra $\boldsymbol{C}\left[D_{n}\right]$ of $D_{n}$ over $\boldsymbol{C}$. Then the Kauffman polynomial of a closed $n$-knit is obtained through $E_{n}(\alpha, \beta)$.

From Section 7 on, we treat the case $n=3$. Then we can show that the function $F$ is a sum of traces of irreducible representations of the algebra $E_{3}(\alpha, \beta)$ (Theorem 10.1). The author expects the same is true for general $n$. In Sections 12-16, we apply our formula to closed 3-braids and 2-bridge links, since they are special types of closed 3-knits. For example, if two closed 3braids have the same writhe (or twist number) and the same Jones polynomial, they also have the same $Q$-polynomial (Theorem 13.1). Thus for the closed 3-braid, the Alexnader polynomial and the writhe determine the two variable Jones, Jones, and $Q$-polynomials. In the actual claculation of the examples in Sections 14 b), c) and 16, the author used a personal computer (NEC PC-9801) with muMATH-83 (Symbolic Mathematics Package) for MS-DOS.

Acknowledgments. I am profoundly indebted to A. Gyoja who gave me many informations about Iwahori algebras. As well, the notion of knit semi-

* This research was supported in part by the Grant in Aid for Scientific Research, the Mintisry of Education, Science and Culture. 
groups arose in discussions with him. I would like to thank T. Kanenobu who introduced me the statement of Theorem 13.1 holds for every example he calculated.

2. The Kauffman polynomial and the $L$-polynomial. Fix non-zero complex numbers $\alpha, \beta$. Let $L(\alpha, \beta, \cdot)$ be the $L$-polynomial [11] which is a regular isotopy invariant of unoriented link diagrams defined by following relations (2.1), (2.2) and (2.3):

$$
\begin{gathered}
L(\alpha, \beta, O)=1 \text { for a planar circle } O \\
L\left(\alpha, \beta,\left|K_{+}\right|\right)+L\left(\alpha, \beta,\left|K_{-}\right|\right) \\
=\left(\beta+\beta^{-1}\right)\left(L\left(\alpha, \beta,\left|K_{0}\right|\right)+L\left(\alpha, \beta,\left|K_{\infty}\right|\right)\right), \\
L\left(\alpha, \beta,\left|K_{l+}\right|\right)=\alpha L\left(\alpha, \beta,\left|K_{\sim}\right|\right) \\
L\left(\alpha, \beta,\left|K_{l-}\right|\right)=\alpha^{-1} L\left(\alpha, \beta,\left|K_{\sim}\right|\right),
\end{gathered}
$$

where $\left|K_{*}\right|$ 's are unoriented link diagrams, identical without a circle where they are as in Figures 1 and 2.

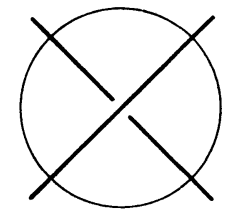

$K_{+}$

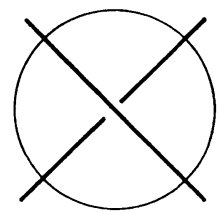

K.

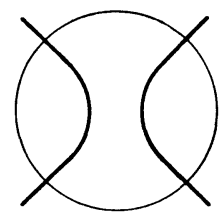

$K_{0}$

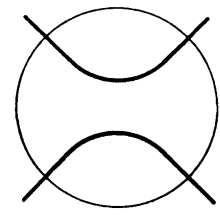

$K_{\infty}$

Figure 1

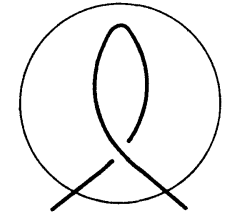

$K_{l+}$

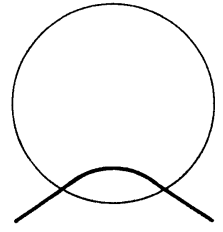

K

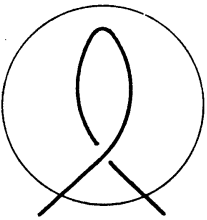

$K_{l-}$

Figure 2

For an oriented link $K$, the writhe (or twist number) $w(K)$ is the sum of the signs of all crossings. The sign of a crossing point is defined as in Figure 3. The Kauffman polynomial $F(\alpha, \beta, K)$ [12] for an oriented link diagram $K$ is defined by: 


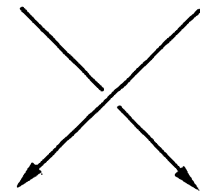

$\operatorname{sign}=+1$

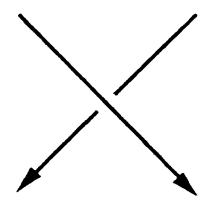

$\operatorname{sign}=-1$

Figure 3

$$
F(\alpha, \beta, K)=\alpha^{-w(K)} L(\alpha, \beta,|K|),
$$

where $|K|$ is an unoriented link coming from $K$ in disregard of its orientation. Then it is an ambient isotopy invariant and is an invariant of link isotopy types.

$\sigma_{i}$

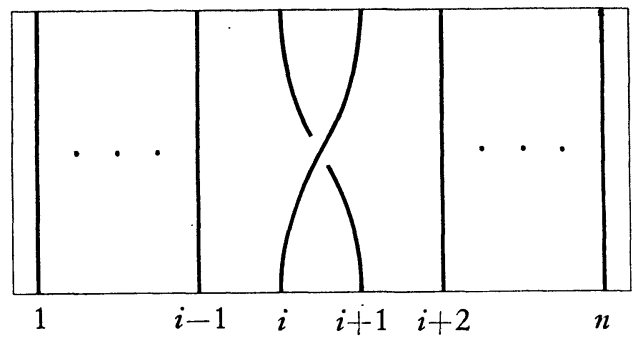

$\sigma_{i}^{-1}$
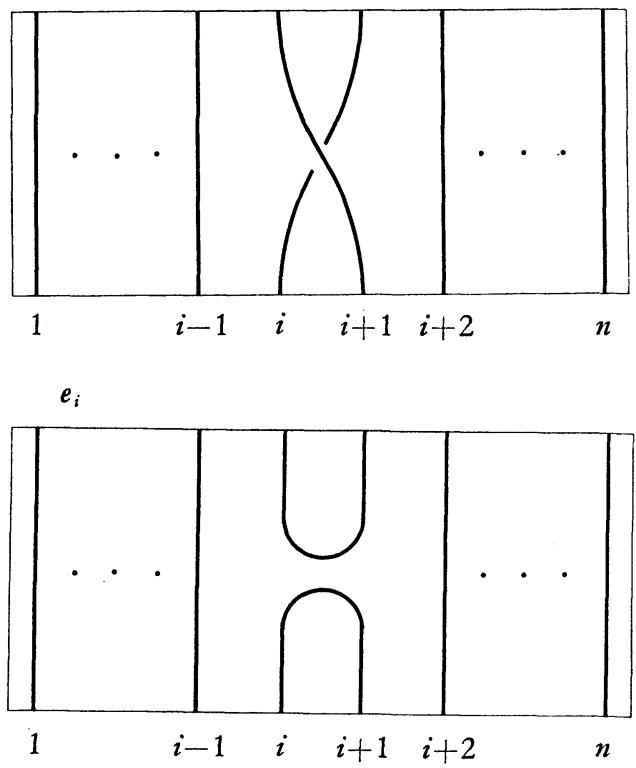

Figure 4 
3. Rectangular diagrams. Fix a positive integer $n$. Let $G_{n}=\left\{\sigma_{i}, \sigma_{i}^{-1}\right.$, $\left.e_{i} \mid i=1,2, \cdots, n-1\right\}$. Let $F_{n}$ be a free semi-group with identity element 1 generated by $G_{n}$. Then $F_{n}=\left\{w_{1} w_{2} \cdots w_{r} \mid r \in N, w_{i} \in G_{n}\right\} \cup\{1\}$. A rectangular diagram $R(w)$ corresponding to $w \in G_{n}$ are defined as in Figure 4. A rectangular diagram $R(w)$ corresponding to $w \in F_{n}$ is defined as in Figure 5 .

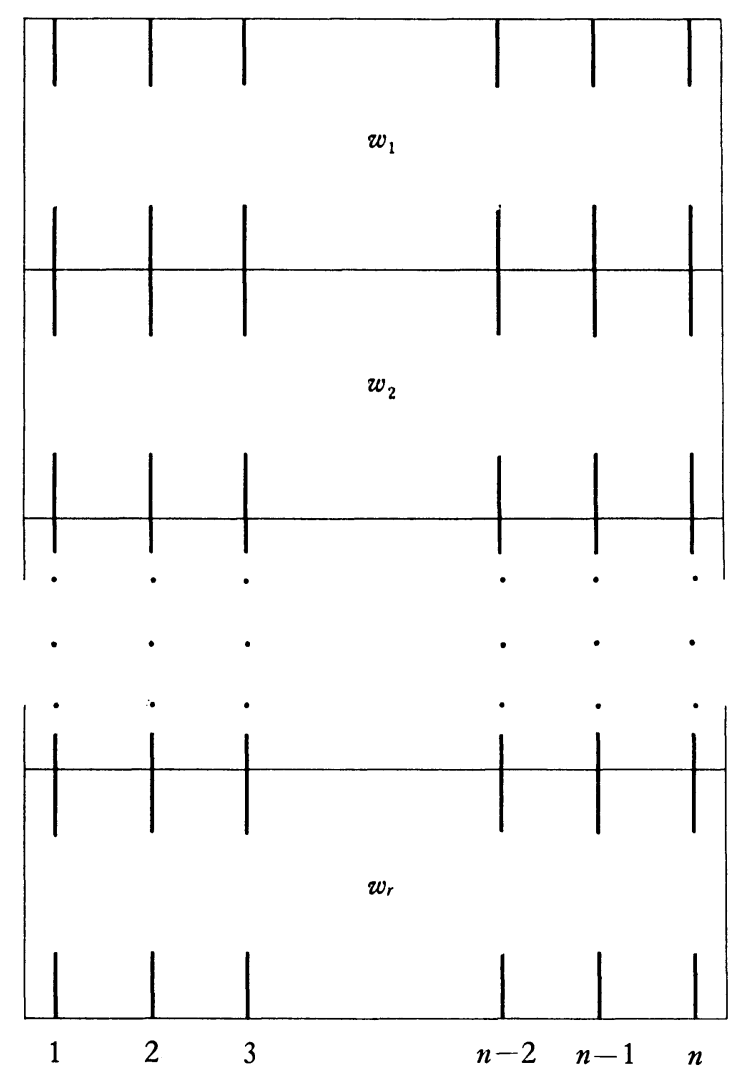

Figure 5

As in Figure 6, we get an unoriented link diagram $w^{\wedge}$ associated with $w \in F_{n}$, connecting upper end points and lower end points of a rectangular diagram $R(w)$.

4. Basic deformations. The three basic deformations of rectangular diagrams are as shown in Figure 7. It shows representative situations for each deformation. If a rectangular diagram has the local forms as shown in this figure, the deformation is performed without disturbing the rest of the diagram. 


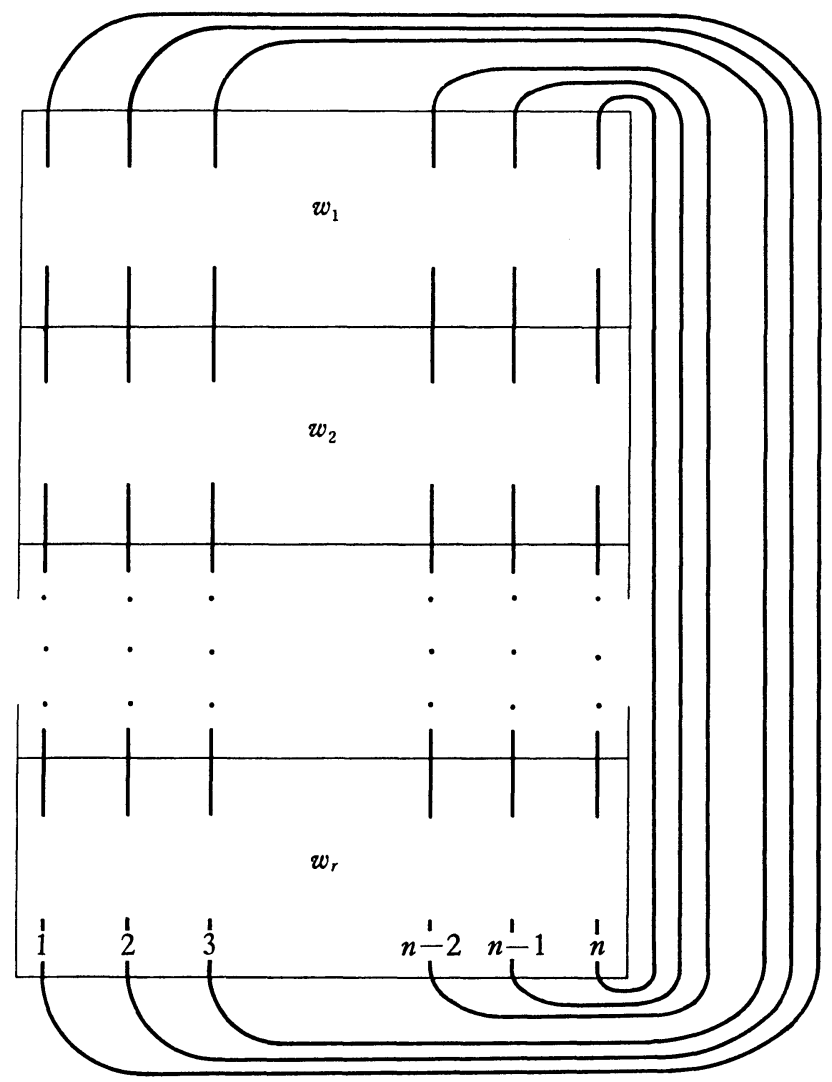

Figure 6

Two rectangular diagrams $R$ and $R^{\prime}$ are called regular isotopic iff there is a sequence of basic deformations of types II and III carring $R$ to $R^{\prime}$. They are called ambient isotopic iff there is a sequence of basic deformations of types I, II and III carring $R$ to $R^{\prime}$.

\section{Knit semi-groups. Let}

$$
D_{n}=F_{n} /\left(w=w^{\prime} \text {, if } R(w) \text { and } R\left(w^{\prime}\right)\right. \text { are regular isotopic). }
$$

We call $D_{n}$ a knit semi-group of degree $n$ and call its element an $n$-knit or simply a knit. For an $n$-knit $b$, we denote by $R(b)$ the regular isotopy class of the rectangular diagram associated to $b$. We denote by $b^{\wedge}$ the set of link diagrams obtained by closing the rectangular diagrams associated with the elements of $F_{n}$ contained in the class of $b$. We call $b^{\wedge}$ the closed $n$-knit coming from $b$.

Proposition 5.2. The L-polynomial may be considered as a map from $D_{n}$ to 
type I

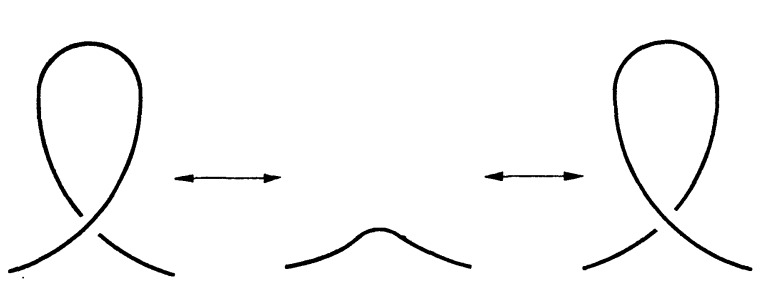

type II

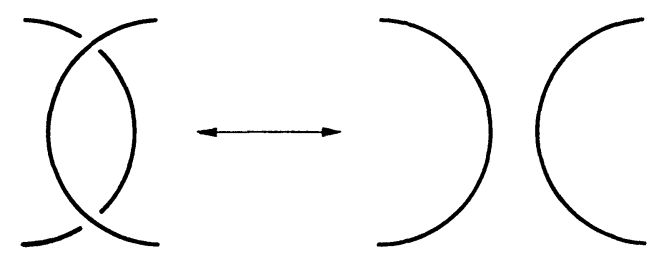

type III
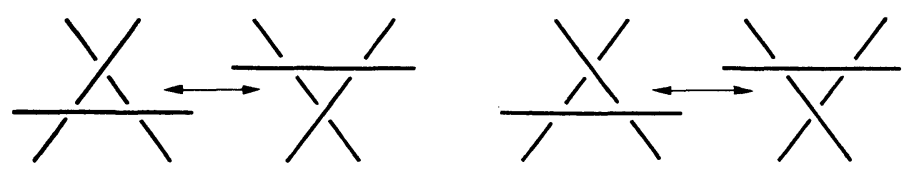

Figure 7

C. We denote it by $L_{D}(\alpha, \beta, \cdot)$.

Proof. For $x \in D_{n}$, let $L_{D}(\alpha, \beta, x)=L\left(\alpha, \beta, \bar{x}^{\wedge}\right)$ where $x$ is a representative of $x$ in $F_{n}$. Because the $L$-polynomial is a regular isotopy invariant, $L_{D}(\alpha, \beta, x)$ does not depend on the choice of $x$.

6. An algebra associated with the $L$-polynomial. The writhe (or twist number) of a string (or component) of a rectangular diagram is the sum of the signs of all the crossings of this string with itself. There are two ways to give an orientation to the string. But the writhe of the string does not depend on the choice of it. The writhe of a rectangular diagram $R$ is the sum of the writhe of all strings of it. We denote it $w(R)$. It is invariant under the regular isotopy. Hence we may define the writhe of a knit $x \in D_{n}$ by $w(x)=$ $w(R(x))$. For $x, y \in D_{n}$, we denote $x \sim y$ if $R(x)$ and $R(y)$ are ambient isosopic. Let

$$
\begin{array}{r}
E_{n}(\alpha, \beta)=C\left[D_{n}\right] /\left(\sigma_{i}+\sigma_{i}^{-1}-\left(\beta+\beta^{-1}\right)\left(1+e_{i}\right) \text { for } 1 \leqq i \leqq n-1,\right. \\
\left.\alpha^{-w(x)} x-\alpha^{-w(y)} y \text { for } x, y \in D_{n} \text { s.t. } x \sim y\right),
\end{array}
$$


where $C\left[D_{n}\right]$ is the semi-group algebra of $D_{n}$.

Proposition 6.2. There is a map $L_{E}(\alpha, \beta, \cdot): E_{n}(\alpha, \beta) \rightarrow C$ commuting with the map $L_{D}(\alpha, \beta, \cdot): D_{n} \rightarrow C$ and the natural map $D_{n} \rightarrow E_{n}(\alpha, \beta)$.

Proof. It is an easy consequence of the definition of the $L$-polynomial given in Section 2.

7. A candidate $\bar{E}_{3}(\alpha, \beta)$ for $E_{3}(\alpha, \beta)$. In the following in this paper, we will restrict ourselves to the case $n=3$. In this section we will define an algebra $\bar{E}_{3}(\alpha, \beta)$ which is a candidate for $E_{3}(\alpha, \beta)$. Let

$$
E_{3}(\alpha, \beta)=C\left[F_{3}\right] / \bar{J}
$$

where $\bar{J}$ is a two sided ideal generated by $J_{1} \cup J_{2} ; J_{1}=\left\{\sigma_{1} \sigma_{1}^{-1}-1, \sigma_{1}^{-1} \sigma_{1}-1\right.$, $\sigma_{2} \sigma_{2}^{-1}-1, \sigma_{2}^{-1} \sigma_{2}-1, \sigma_{1} \sigma_{2} \sigma_{1}-\sigma_{2} \sigma_{1} \sigma_{2}, e_{1} e_{2} e_{1}-e_{1}, e_{2} e_{1} e_{2}-e_{2}, \sigma_{1} \sigma_{2} e_{1}-e_{2} e_{1}, \sigma_{2} \sigma_{1} e_{2}-e_{1} e_{2}$, $\left.\sigma_{1} e_{2} \sigma_{1}^{-1}-\sigma_{2}^{-1} e_{1} \sigma_{2}, \sigma_{2} e_{1} \sigma_{2}^{-1}-\sigma_{1}^{-1} e_{2} \sigma_{1}\right\}, J_{2}=\left\{\alpha \sigma_{1} e_{1}-e_{1}, \alpha e_{1} \sigma_{1}-e_{1}, \alpha \sigma_{2} e_{2}-e_{2}, \alpha e_{2} \sigma_{2}-e_{2}\right.$, $\left.\alpha^{-1} e_{1} \sigma_{2} e_{1}-e_{1}, \alpha^{-1} e_{2} \sigma_{1} e_{2}-e_{2}, \sigma_{1}+\sigma_{1}^{-1}-\left(\beta+\beta^{-1}\right)\left(1+e_{1}\right), \sigma_{2}+\sigma_{2}^{-1}-\left(\beta+\beta^{-1}\right)\left(1+e_{2}\right)\right\}$.

Proposition 7.2. There exists a natural surjective homomorphism $\varphi: \bar{E}_{3}(\alpha, \beta)$ $\rightarrow E_{3}(\alpha, \beta)$.

Proof. Let $p_{1}: \boldsymbol{C}\left[F_{3}\right] \rightarrow \bar{E}_{3}(\alpha, \beta)$ and $p_{2}: \boldsymbol{C}\left[F_{3}\right] \rightarrow E_{3}(\alpha, \beta)$ be the natural homomorphisms. To show the existence of a surjective homomorphism from $E_{3}(\alpha, \beta)$ to $E_{3}(\alpha, \beta)$ commuting with $p_{1}$ and $p_{2}$, we must check that $\operatorname{ker} p_{2} \supset$ $\operatorname{ker} p_{1}=\bar{J}$. Because the two terms of elements of $J_{1}$ are regular isotopic, they are contained in ker $p_{2}$ according to the definition (5.1) of $D_{n}$. Because of the definition (6.1) of $E_{3}(\alpha, \beta)$, the elements of $J_{2}$ are all contained in $\operatorname{ker} p_{2}$. Hence any element of $\bar{J}$ is contained in $\operatorname{ker} p_{2}$ and Proposition 7.2 is proved.

\section{Structure of $\bar{E}_{3}(\alpha, \beta)$}

Proposition 8.1. $\quad \bar{E}_{3}(\alpha, \beta) \cong M_{3}(\boldsymbol{C})+\boldsymbol{C}+M_{2}(\boldsymbol{C})+\boldsymbol{C}$ for generic $\alpha, \beta$.

Proof. A basis of $\bar{E}_{3}(\alpha, \beta)$ over $\boldsymbol{C}$ is $V_{0}=\left\{1, \sigma_{1}, \sigma_{2}, \sigma_{1} \sigma_{2}, \sigma_{2} \sigma_{1}, \sigma_{1} \sigma_{2} \sigma_{1}, e_{1}, \sigma_{2} e_{1}\right.$, $\left.e_{2} e_{1}, e_{2}, \sigma_{1} e_{2}, e_{1} e_{2}, \sigma_{1} e_{2} \sigma_{1}, e_{1} \sigma_{2}, e_{2} \sigma_{1}\right\} . \quad$ Let $I(\alpha, \beta)$ be the two sided ideal of $\bar{E}_{3}(\alpha, \beta)$ generated by $e_{1}$. Then a bisis of $I(\alpha, \beta)$ is $\left\{e_{1}, \sigma_{2} e_{1}, e_{2} e_{1}, e_{2}, \sigma_{1} e_{2}, e_{1} e_{2}, e_{1} \sigma_{2}, e_{2} \sigma_{1}\right.$, $\left.\sigma_{1} e_{2} \sigma_{1}\right\}$ and $I(\alpha, \beta)$ is isomorphic to $M_{3}(C)$ as an algebra for generic $\alpha, \beta$. We also have $\bar{E}_{3}(\alpha, \beta) / I(\alpha, \beta) \cong H_{3}(\beta)$ where $H_{3}(\beta)=\boldsymbol{C}\left\langle\sigma_{1}, \sigma_{2}\right\rangle /\left(\sigma_{i}+\sigma_{i}^{-1}-\left(\beta+\beta^{-1}\right)\right.$. $\left.1(i=1,2), \sigma_{1} \sigma_{2} \sigma_{1}-\sigma_{2} \sigma_{1} \sigma_{2}\right)$, which is the Iwahori algebra ([2], Chapt. IV, §2, Ex. 22-25) associated with the symmetric group $S_{3}$ of degree 3 . Except for finite numbers of $\beta, H_{3}(\beta)$ is known to be completely reducible and isomorphic to the group ring of $S_{3}: H_{3}(\beta) \cong \boldsymbol{C}\left[S_{3}\right] \cong \boldsymbol{C}+M_{2}(\boldsymbol{C})+\boldsymbol{C}$. We also know that $\operatorname{dim}_{\boldsymbol{C}} H_{3}(\beta)$ 
$+\operatorname{dim}_{C} I(\alpha, \beta)=\operatorname{dim}_{C} \bar{E}_{3}(\alpha, \beta)$ because $\operatorname{dim}_{C} H_{3}(\beta)=6, \operatorname{dim}_{C} I(\alpha, \beta)=9$ and $\operatorname{dim}_{C} E_{3}(\alpha, \beta)=15$. Hence the cannonical projection $E_{3}(\alpha, \beta) \rightarrow H_{3}(\beta)$ splits and we get the proposition.

In the following of this paper, fix generic $\alpha, \beta \in \boldsymbol{C}-\{0\}$ so that Proposition 8.1 holds.

9. Irreducible representations of $D_{3}$ through $E_{3}(\alpha, \beta)$. As shown in Proposition 8.1, $\bar{E}_{3}(\alpha, \beta)$ consists of four simple algebras. Let $p_{0}, p_{1}, p_{2}, p_{3}$, be the cannonical projections from $\bar{E}_{3}(\alpha, \beta)$ to its irreducible components $M_{3}(\boldsymbol{C}), \boldsymbol{C}$, $M_{2}(\boldsymbol{C}), \boldsymbol{C}$, respectively. Then a composition of the cannonical projection $\boldsymbol{C}\left[D_{3}\right]$ $\rightarrow E_{3}(\alpha, \beta)$ and $p_{i}(i=0,1,2,3)$ induces an irreducible linear representations of $D_{3}$, say $\rho_{i}$. Representation matrices of $\rho_{0}, \rho_{1}, \rho_{2}, \rho_{3}$ are given as follows:

$$
\begin{aligned}
& \rho_{0}\left(\sigma_{1}\right)=\left[\begin{array}{crr}
\alpha^{-1} & 0 & z \\
0 & 0 & -1 \\
0 & 1 & z
\end{array}\right], \quad \rho_{0}\left(\sigma_{2}\right)=\left[\begin{array}{ccc}
0 & -1 & \alpha^{-1} \\
1 & z & 0 \\
0 & \alpha^{-1} z & 0
\end{array}\right] \text {, } \\
& \rho_{0}\left(e_{1}\right)=\left[\begin{array}{ccc}
\mu & \alpha & 1 \\
0 & 0 & 0 \\
0 & 0 & 0
\end{array}\right], \quad \rho_{0}\left(e_{2}\right)=\left[\begin{array}{ccc}
0 & 0 & 0 \\
0 & 0 & 0 \\
1 & \alpha & \mu
\end{array}\right], \\
& \rho_{1}\left(\sigma_{1}\right)=\beta, \quad \rho_{1}\left(\sigma_{2}\right)=\beta, \quad \rho_{1}\left(e_{1}\right)=0, \quad \rho_{1}\left(e_{2}\right)=0, \\
& \rho_{2}\left(\sigma_{1}\right)=\left[\begin{array}{cc}
\beta & \sqrt{-1} \\
0 & \beta^{-1}
\end{array}\right], \quad \rho_{2}\left(\sigma_{2}\right)=\left[\begin{array}{cc}
\beta^{-1} & 0 \\
\sqrt{-1} & \beta
\end{array}\right], \quad \rho_{2}\left(e_{1}\right)=\rho_{2}\left(e_{2}\right)=\left[\begin{array}{ll}
0 & 0 \\
0 & 0
\end{array}\right], \\
& \rho_{3}\left(\sigma_{1}\right)=\beta^{-1}, \quad \rho_{3}\left(\sigma_{2}\right)=\beta^{-1}, \quad \rho_{3}\left(e_{1}\right)=0, \quad \rho_{3}\left(e_{2}\right)=0,
\end{aligned}
$$

where $z=\beta+\beta^{-1}$ and $\mu=\left(\alpha+\alpha^{-1}\right) / z-1$. The matrices of $\rho_{1}, \rho_{2}, \rho_{3}$ are obtained from that of irreducible representations of the Iwahori algebra associated with $S_{3}$. Let $W$ be a subspace of $E_{3}(\alpha, \beta)$ spanned by $e_{1}, \sigma_{2} e_{1}, e_{2} e_{1}$ then $W$ is invariant under the left action of $E_{3}(\alpha, \beta)$. The matrix representations of the restrictions of $\rho_{0}\left(\sigma_{i}\right), \sigma_{0}\left(e_{i}\right)(i=1,2)$ on $W$ with respect to the basis $\left\{e_{1}, \sigma_{2} e_{1}, e_{2} e_{1}\right\}$ are as above. For example, $\sigma_{1} e_{1}=\alpha^{-1} e_{1}, \sigma_{1} e_{2} e_{1}=\sigma_{2} e_{1}, \sigma_{1} e_{2} e_{1}=\sigma_{2}^{-1} e_{1}=-\sigma_{2} e_{1}+z e_{2} e_{1}+z e_{1}$ and so we have the matrix of $\rho_{0}\left(\sigma_{1}\right)$.

10. Formulas for the $L$-polynomial, the Kauffman polynomial and the Q-polynomial of a closed 3-knit

Theorem 10.1. Let $K$ be an oriented link diagram such that the unoriented link diagram $|K|$ coming from $K$ in disregard of its orientation is euqal to a closed 
3-knit $x^{\wedge}$ associated with $x \in D_{3}$. Let $\chi_{0}, \chi_{1}, \chi_{2}, \chi_{3}$ be the traces of irreducible representations $\rho_{1}, \rho_{2}, \rho_{3}, \rho_{4}$ of $D_{3}$ defined in Section 9 . Then we have

$$
\begin{aligned}
& L(\alpha, \beta,|K|)=\chi_{0}(x)+a_{1} \chi_{1}(x)+a_{2} \chi_{2}(x)+a_{3} \chi_{3}(x), \\
& F(\alpha, \beta, K)=\alpha^{-w(K)}\left(\chi_{0}(x)+a_{1} \chi_{1}(x)+a_{2} \chi_{2}(x)+a_{3} \chi_{3}(x)\right),
\end{aligned}
$$

where $w(K)$ is the writhe of $K$ defined in Section 2 and $a_{1}, a_{2}, a_{3}$ are given as follows:

$$
\begin{aligned}
& a_{1}=P\left(\beta^{-3}\left(\mu^{2}-3\right)+\left(1-2 \beta^{-2}\right)\left(\alpha \mu-z-\alpha^{-1}\right)+\left(\beta^{-1}-\beta\right) \alpha^{2}\right), \\
& a_{2}=P\left(\left(\beta-\beta^{-1}\right)\left(\mu^{2}-3\right)+\left(\beta^{-2}-\beta^{2}\right)\left(\alpha \mu-z-\alpha^{-1}\right)+\left(\beta-\beta^{-1}\right) \alpha^{2}\right), \\
& a_{3}=P\left(-\beta^{3}\left(\mu^{2}-3\right)+\left(2 \beta^{2}-1\right)\left(\alpha \mu-z-\alpha^{-1}\right)+\left(\beta^{-1}-\beta\right) \alpha^{2}\right),
\end{aligned}
$$

where $z=\beta+\beta^{-1}, \mu=\left(\alpha+\alpha^{-1}\right) / z-1$ and $P=1 /\left(\beta^{-1}-\beta\right)\left(\beta^{2}-1+\beta^{-2}\right)$.

The $Q$-polynomial (or absolute polynomial) of an unoriented link $|K|$ is defined by $Q(\beta,|K|)=L(1, \beta,|K|)$, which is an invariant of isotopy types $[3],[6]$.

Corollary 10.5. Let $K$ and $x$ be as in Theorem 10.1. Then we have

$$
\begin{aligned}
Q(\beta,|K|) & =\chi_{0}(x)+\frac{2 z^{2}+2 z-2}{z^{2}\left(z^{2}-3\right)}\left(\chi_{1}(x)+\chi_{3}(x)\right) \\
& +\frac{-z^{4}-2 z^{3}+3 z^{2}+4 z-4}{z^{2}\left(z^{2}-3\right)} x_{2}(x) .
\end{aligned}
$$

Proof of Theorem 10.1. According to Propositions 5.2 and 6.2, we already know that $L(\alpha, \beta,|K|)=L_{D}(\alpha, \beta, x)=L_{E}(\alpha, \beta, \varphi(p(x)))$. Because of the definition of the $L$-polynomial, we have $L_{E}(\alpha, \beta, \varphi(p(x)))=L_{\bar{E}}\left(\alpha, \beta, \varphi\left(p\left(g x g^{-1}\right)\right)\right)$ for $x \in D_{3}$ and $g \in B_{3} \subset D_{3}$. But $p\left(B_{3}\right)$ generates $E_{3}(\alpha, \beta)$ as a $C$-algebra and $E_{3}(\alpha, \beta)$ is semi-simple, and so $L_{E}(\alpha, \beta, \varphi(p(x)))$ is the sum of traces of irreducible representations of $D_{3}$ coming from the irreducible components of $E_{3}(\alpha, \beta)$ treated in Section 9, i.e. $L_{E}(\alpha, \beta, \varphi(p(x)))=a_{0} \chi_{0}(x)+a_{1} \chi_{1}(x)+a_{2} \chi_{2}(x)+a_{3} \chi_{3}(x)$. But the values of $\chi_{0}, \chi_{1}, \chi_{2}, \chi_{3}$ and $L_{D}(\alpha, \beta, \cdot)$ for $1, \sigma_{1}, \sigma_{1} \sigma_{2}, e_{1}$ in $D_{3}$ are given as follows:

\begin{tabular}{c|cccc|c}
$D_{3}$ & $\chi_{0}$ & $\chi_{1}$ & $\chi_{2}$ & $\chi_{3}$ & $L_{D}(\alpha, \beta, \cdot)$ \\
\hline 1 & 3 & 1 & 2 & 1 & $\mu^{2}$ \\
$\sigma_{1}$ & $\beta+\beta^{-1}+\alpha^{-1}$ & $\beta$ & $\beta+\beta^{-1}$ & $\beta^{-1}$ & $\alpha \mu$ \\
$\sigma_{1} \sigma_{2}$ & 0 & $\beta^{2}$ & 1 & $\beta^{-2}$ & $\alpha^{2}$ \\
$e_{1}$ & $\mu$ & 0 & 0 & 0 & $\mu$ \\
\hline
\end{tabular}


Hence we have $a_{0}=1$ and $a_{1}, a_{2}, a_{3}$ as in (10.4).

11. $E_{3}(\alpha, \beta)$ is isomorphic to $E_{3}(\alpha, \beta)$

Corollary 11.1. The cannonical projection $\varphi: E_{3}(\alpha, \beta) \rightarrow E_{3}(\alpha, \beta)$ defined in Proposition 7.2 is an isomorphism for generic $\alpha, \beta$.

Proof. Assume that $\operatorname{ker} \varphi \supsetneq\{0\}$. Then $\operatorname{ker} \varphi$ contains at least one irreducible component of $\bar{E}_{3}(\alpha, \beta)$. But in this situation, the coefficient $a_{i}$ in (10.5) corresponding ot above irreducible component must be zero. But $a_{0}=1$ and $a_{1}, a_{2}, a_{3}$ are all non-zero for generic $\alpha, \beta$ according to (10.4). It contradicts the assumption and we get $\operatorname{ker} \varphi=\{0\}$. Hence $\varphi$ is an isomorphism.

12. The Kauffman polynomials of closed 3-braids. Let $B_{3}$ be a braid group on three strings. $B_{3}$ is contained in $D_{3}$ as a sub-semigroup. Let $b$ be a 3 -braid and $b^{\wedge}$ be its closed braid, then the writhe $w\left(b^{\wedge}\right)$ is equal to the exponent $\operatorname{sum} \varepsilon(b)$ of $b$. Let

$$
C=\left\{\left(b, b^{\prime}\right) \in B_{3} \times B_{3} \mid V(\hat{b})=V\left(\hat{b}^{\prime}\right), \varepsilon(b)=\varepsilon\left(b^{\prime}\right)\right\}
$$

where $V:\{$ link $\} \rightarrow \boldsymbol{C}\left(t^{1 / 2}\right)$ is the Jones invariant of links.

Theorem 12.2. Let $\left(b_{1}, b_{2}\right)$ be a pair of 3-braids in $C$. Then $F\left(\alpha, \beta, b_{1}{ }^{\wedge}\right)=$ $F\left(\alpha, \beta, b_{2}{ }^{\wedge}\right)$ iff $\chi_{0}\left(b_{1}\right)=\chi_{0}\left(b_{2}\right)$.

Proof. For $\left(b_{1}, b_{2}\right)$ in $C, w\left(b_{1}{ }^{\wedge}\right)=w\left(b_{2}{ }^{\wedge}\right)$ and $\chi_{i}\left(b_{1}\right)=\chi_{i}\left(b_{1}\right)$ for $i=1,3$ since $\varepsilon\left(b_{1}\right)=\varepsilon\left(b_{2}\right)$. Because

$$
\beta \cdot P^{-1} \rho_{2}\left(\sigma_{1}\right) P=\left[\begin{array}{rr}
-t & 1 \\
0 & 1
\end{array}\right], \quad \beta \cdot P^{-1} \rho_{2}\left(\sigma_{2}\right) P=\left[\begin{array}{rr}
1 & 0 \\
t & -t
\end{array}\right],
$$

where $t=-\beta^{2}$ and $P=\left[\begin{array}{cc}\sqrt{-1} \cdot \beta & 0 \\ 0 & 1\end{array}\right]$, we have $\chi_{2}\left(b_{1}\right)=\chi_{2}\left(b_{2}\right)$ as shown in [1]. Using (10.3), we get $F\left(\alpha, \beta, b_{1}^{\wedge}\right)-F\left(\alpha, \beta, b_{2}{ }^{\wedge}\right)=\alpha^{-w\left(b_{1}\right)}\left(\chi_{0}\left(b_{1}\right)-\chi_{0}\left(b_{2}\right)\right)$. This proves Theorem 12.2.

\section{The Q-polynomial of closed 3-braids}

Theorem 13.1. Let $C$ be the subset of $B_{3} \times B_{3}$ defined in Section 12. Then for $\left(b, b^{\prime}\right) \in C$, we have $Q(\beta, \hat{b})=Q\left(\beta, \hat{b}^{\prime}\right)$.

Therefore, we have, from [1], [8] and [9],

Corollary 13.2. For the closed 3-braid, the two variable Jones, Jones, and $Q$-polynomials are determined by the Alexander polynomial and the writhe. 
Proof of Theorem 13.1. Let $\rho_{0}, \rho_{2}$ be the representations of $D_{3}$ and $\chi_{0}, \chi_{2}$ be their traces defined in Section 9. We also denote $\rho_{0}(y)=\rho_{0}(\alpha, \beta, y), \chi_{0}(y)=$ $\chi_{0}(\alpha, \beta, y), \rho_{2}(y)=\rho_{2}(\beta, y)$ and $\chi_{2}(y)=\chi_{2}(\beta, y)$ for a 3-knit $y$ if the parameters $\alpha, \beta$ are needed. Because of Theorem 12.2 and $Q(\beta, \hat{b})=F(1, \beta, \hat{b})$, we get $Q(\beta, \hat{b})-Q\left(\beta, \hat{b}^{\prime}\right)=\chi_{0}(1, \beta, b)-\chi_{0}\left(1, \beta, b^{\prime}\right)$. Let

$$
P=\left[\begin{array}{ccc}
1 & -1 / \beta & 0 \\
0 & 1 & 0 \\
0 & -\beta & 1
\end{array}\right]\left[\begin{array}{ccc}
\sqrt{-1} & 0 & 0 \\
-\sqrt{-1} \cdot B_{1} B_{3} & B_{3}-\sqrt{-1} \cdot B_{1} B_{3} \\
0 & 0 & -\sqrt{-1}
\end{array}\right]
$$

where $B_{1}=\beta^{1 / 2}-\beta^{-1 / 2}$ and $B_{3}=1 /\left(\beta^{3 / 2}+\beta^{-3 / 2}\right)$. Then we have

$$
\begin{aligned}
& P^{-1} \rho_{0}\left(1, \beta, \sigma_{1}\right) P=\left[\begin{array}{cccc}
\beta & \sqrt{-1} \cdot \beta^{1 / 2} & -1 \\
0 & 1 & 2 \sqrt{-1} \cdot \beta^{-1 / 2} \\
0 & 0 & \beta^{-1}
\end{array}\right], \\
& P^{-1} \rho_{0}\left(1, \beta, \sigma_{2}\right) P=\left[\begin{array}{ccc}
\beta^{-1} & 0 & 0 \\
2 \sqrt{-1} \cdot \beta^{-1 / 2} & 1 & 0 \\
-1 & \sqrt{-1} \cdot \beta^{1 / 2} & \beta
\end{array}\right] .
\end{aligned}
$$

The representation matrices of $\sigma_{1}$ and $\sigma_{2}$ of the symmetric tensor of degree 2 of $\rho_{2}(\beta, \cdot)$, say $\rho_{2}^{(2)}(\beta, \cdot)$, are equal to those of $P^{-1} \rho_{0}\left(1, \beta^{2}, \cdot\right) P$, where the matrix of symmetric tensor of degree 2 of a 2 by 2 matrix is given as follows:

$$
S^{(2)}: A=\left[\begin{array}{ll}
a & b \\
c & d
\end{array}\right] \rightarrow\left[\begin{array}{ccc}
a^{2} & a b & b^{2} \\
2 a c & a d+b c & 2 b d \\
c^{2} & c d & d^{2}
\end{array}\right]
$$

An elementary calculation shows that $S^{(2)}(A B)=S^{(2)}(A) S^{(2)}(B)$ for $A, B \in M_{2}(C)$ and the trace of $S^{(2)}(A)$ is equal to trace $(A)^{2}-\operatorname{det}(A)$. Hence we get $\chi_{0}\left(1, \beta^{2}, \cdot\right)$ $=\chi_{2}(\beta, \cdot)^{2}-1$ and $Q\left(\beta^{2}, \hat{b}\right)=Q\left(\beta^{2}, \hat{b}^{\prime}\right)$. But this equality holds for every generic complex number $\beta$ and so we have $Q(\beta, \hat{b})=Q\left(\beta, \hat{b}^{\prime}\right)$.

14. Examples for Theorem 12.2. In [1], Birman gave examples of pairs of knots coming from closed 3-braids for whihc the Jones invariant does not work well. According to Theorem 13.1, the $Q$-polynomial is not good for them, too. Here we show the Kauffman invariant is good enough for some of them.

a) Let $x=\sigma_{1}^{p_{1}} \sigma_{2}^{q_{1}} \cdots \sigma_{1}^{p_{s}} \sigma_{2}^{q_{s}}\left(p_{i}>0, q_{i}<0\right.$ for $1 \leqq i \leqq s, \varepsilon(x)=p_{1}+\cdots+p_{s}+q_{1}+$ $\left.\cdots+q_{s}=6 r \neq 0\right)$ and $y=\left(\sigma_{1} \sigma_{2} \sigma_{1}\right)^{4 r} x^{-1}$. Then $V(\hat{x})=V(\hat{y})$ and $Q(\beta, \hat{x})=Q(\beta, \hat{y})$ but $F(\alpha, \beta, \hat{x}) \neq F(\alpha, \beta, \hat{y})$ because the highest degree of $\alpha$ in $\chi_{0}(x)$ is equal to $-\left(q_{1}+q_{2}+\cdots+q_{s}\right)$ but that of $\alpha$ in $\chi_{0}(y)$ is equal to $\left(p_{1}+p_{2}+\cdots+p_{s}\right) / 3-2\left(q_{1}+\right.$ $\left.q_{2}+\cdots+q_{s}\right) / 3$ and they are different since $p_{1}+\cdots+p_{s}+q_{1}+\cdots+q_{s} \neq 0$. These 
pairs are examples treated in Proposition 2 of [1].

b) $\delta_{1}=\sigma_{1}^{-3} \sigma_{2}^{6} \sigma_{1}^{-1} \sigma_{2}^{9}, \delta_{2}=\sigma_{1}^{-3} \sigma_{2}^{9}, \delta_{3}=\sigma_{1}^{-2} \sigma_{2}^{3}, \gamma=\delta_{1} \delta_{2} \delta_{3}$ and $\mu=\delta_{1} \delta_{3} \delta_{2}$. Then $V(\hat{\gamma})=V(\hat{\mu})$ and $Q(\beta, \hat{\gamma})=Q(\beta, \hat{\mu})$ but $F(\alpha, \beta, \hat{\gamma}) \neq F(\alpha, \beta, \hat{\mu})$.

c) $\delta_{1}^{\prime}=\sigma_{1}^{-7} \sigma_{2}^{8} \sigma_{1}^{-1} \sigma_{2}^{7}, \delta_{2}^{\prime}=\sigma_{1}^{-7} \sigma_{2}^{7}, \delta_{3}^{\prime}=\sigma_{1}^{-6} \sigma_{2}^{-1}, \gamma^{\prime}=\delta_{1}^{\prime} \delta_{2}^{\prime} \delta_{3}^{\prime}$ and $\mu^{\prime}=\delta_{1}^{\prime} \delta_{3}^{\prime} \delta_{2}^{\prime}$. Then $V\left(\hat{\gamma}^{\prime}\right)=V\left(\hat{\mu}^{\prime}\right)$ and $Q\left(\beta, \hat{\gamma}^{\prime}\right)=Q\left(\beta, \hat{\mu}^{\prime}\right)$ but $F\left(\alpha, \beta, \hat{\gamma}^{\prime}\right) \neq F\left(\alpha, \beta, \hat{\mu}^{\prime}\right)$.

15. The Kauffman polynomials of 2-bridge links. Let $m$ be a positive odd integer. A 2-bridge link $K\left(a_{1}, a_{2}, \cdots, a_{m}\right)$ is an oriented link associated to a closed 3-knit $\left(e_{1} \sigma_{2}^{a} \sigma_{1}^{a} \cdots \sigma_{2}^{a}\right)^{\wedge}$ with an orientation as in Figure 8.

2-bridge link

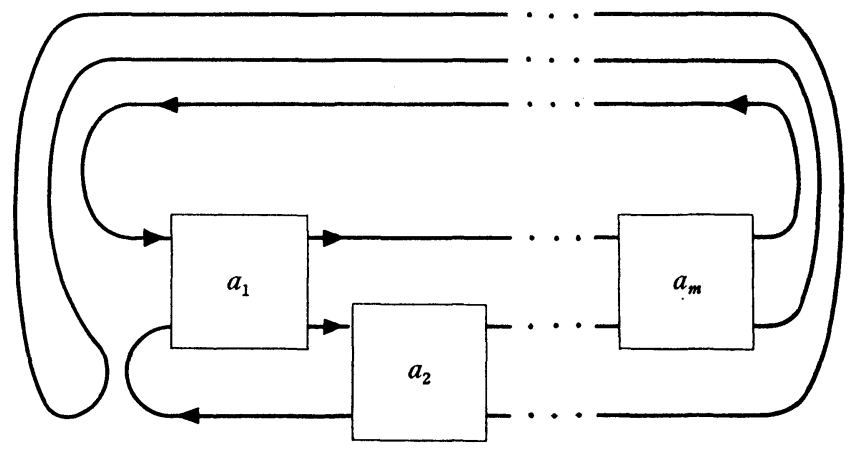

Where

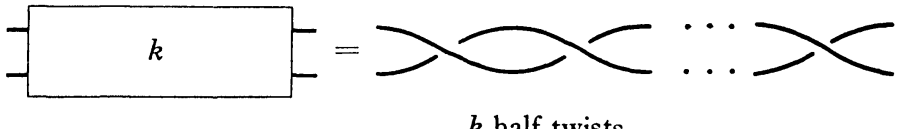

$k$ half twists

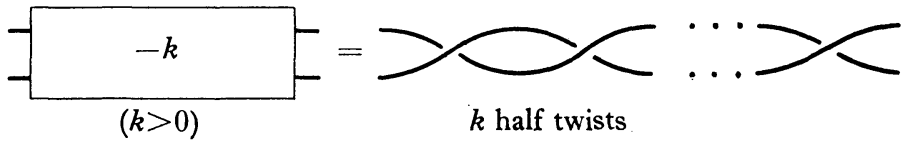

Figure 8

Theorem 15.1. For an oriented 2-bridge link $K=K\left(a_{1}, a_{2}, \cdots, a_{m}\right)$, we have

$$
F(\alpha, \beta, K)=\alpha^{-w(K)} \chi_{0}\left(e_{1} \sigma_{2}^{a} \sigma_{1}^{a} \cdots \sigma_{2}^{a}\right) .
$$

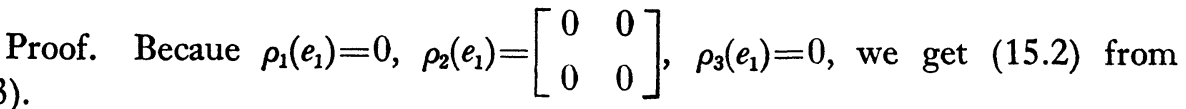


16. Examples for Theorem 15.1. In Theorem 7 of [10], arbitrarily many 2-bridge links with the same 2-variable Alexander, 2-variable Jones and $Q$-polynomials are given. The Kauffman polynomials with specialized values of $\alpha$ and $\beta$ of some of above links are calculated and it turned out that they can be distinguished by the Kauffman polynomials.

Let $\psi$ be a map from invertible elements of $\bar{E}_{3}(\alpha, \beta)$ to $E_{3}(\alpha, \beta)$ defined by $\psi(x)=x \sigma_{1}^{2} x^{-1}$.

a) Let $x_{1}=\sigma_{2}^{2} \sigma_{1}^{2} \sigma_{2}^{2} \sigma_{1}^{-2} \sigma_{2}^{-2}, y_{1}=\sigma_{2}^{-2} \sigma_{1}^{-2} \sigma_{2}^{2} \sigma_{1}^{2} \sigma_{2}^{2}, x_{2}=\psi\left(x_{1}\right), y_{2}=\psi\left(y_{1}\right), z_{1}=$ $e_{1} x_{2}, z_{2}=e_{1} y_{2}$. Let $K_{1}, K_{2}$ be oriented links associated with the closed 3-knits $z_{1}{ }^{\wedge}$ and $z_{2}{ }^{\wedge}$ with orientations as in Figure 8 in Section 15 . Then $K_{1}$ and $K_{2}$ have the same 2-variable Alexander, 2-variable Jones and $Q$-polynomials but have distinct Kauffman polynomials.

b) Let $x_{3}=\psi\left(x_{1}^{-1}\right), y_{3}=\psi\left(y_{1}^{-1}\right), x_{4}=\psi\left(x_{2}\right), y_{4}=\psi\left(y_{2}\right), x_{5}=\psi\left(x_{3}\right), y_{5}=\psi\left(y_{3}\right)$, $z_{3}=e_{1} x_{4}, z_{4}=e_{1} y_{4}, z_{5}=e_{1} x_{5}, z_{6}=e_{1} y_{5}$. Let $K_{3}, K_{4}, K_{5}, K_{6}$ be oriented links associated with the closed 3 -knits $z_{3} \hat{}, z_{4}, z_{5} \hat{}, z_{6} \hat{}$ with orientation as in Figure 8 in Section 15. Then $K_{3}, K_{4}, K_{5}, K_{6}$ have the same 2-variable Alexander, 2variable Jones and $Q$-polynomials but have distinct Kauffman polynomials.

c) Let $x_{6}=\psi\left(x_{2}^{-1}\right), y_{6}=\psi\left(y_{2}^{-1}\right), x_{7}=\psi\left(x_{3}^{-1}\right), y_{7}=\psi\left(y_{3}^{-1}\right), x_{8}=\psi\left(x_{4}\right), y_{8}=$ $\psi\left(y_{4}\right), x_{9}=\psi\left(x_{5}\right), y_{9}=\psi\left(y_{5}\right), x_{10}=\psi\left(x_{6}\right), y_{10}=\psi\left(y_{6}\right), x_{11}=\psi\left(x_{7}\right), y_{11}=\psi\left(y_{7}\right), z_{7}=$ $e_{1} x_{8}, z_{8}=e_{1} y_{8}, z_{9}=e_{1} x_{9}, z_{10}=e_{1} y_{9}, z_{11}=e_{1} x_{10}, z_{12}=e_{1} y_{10}, z_{13}=e_{1} x_{11}, z_{14}=e_{1} y_{11}$. Let $K_{i}(i=7,8, \cdots, 14)$ be an oriented link associated with the closed knit $z_{i}{ }^{\wedge}$ with an orientation as in Figure 8 in Section 14. Then $K_{7}, K_{8}, \cdots, K_{14}$ have the same 2-variable Alexander, 2-variable Jones and $Q$-polynomials but have distinct Kauffman polynomials.

\section{References}

[1] J.S. Birman: On the Jones polynomial of closed 3-braids, Invent. Math. 81 (1985), 287-294.

[2] N. Bourbaki: Eléments de mathématique, groupes et algèbres de Lie, Chapitres 4, 5 et 6. Hermann, Paris, 1968.

[3] R.D. Brandt, W.B.R. Lickorish, K.C. Millett: A polynomial invariant for unoriented knots and links, Invent. Math. 84 (1986), 563-573.

[4] P. Freyd and D. Yetter, J. Hoste, W.B.R. Lickorish and K.C. Millett, A. Ocneanu: A new polynomial invariant of knots and lindks, Bull. Amer. Math. Soc. 12 (1985), 239-246.

[5] A. Gyoja: Topological invariants of links and representations of Hecke algebras II, preprint, Osaka University.

[6] C.F. Ho: A new polynomial invariant for knots and links, preliminary report, Abstracts Amer. Math. Soc. 6 (1985), 300.

[7] N. Iwahori: On the structure of the Hecke ring of a Chevalley group over a finite field, J. Fac. Sci. Univ. Tokyo 10 (part 2), (1964), 215-236. 
[8] V.F.R. Jones: A polynomial invariant for knots via von Neumann algebras, Bull. Amer. Math. Soc. 12 (1985), 103-111.

[9] V.F.R. Jones: Hecke algebra representations of braid groups and link polynomials, Ann. of Math. 126 (1987), 335-388.

[10] T. Kanenobu: Examples on polynomial invariants of knots and links, preprint.

[11] L.H. Kauffman: State models for knot polynomials, preprint.

[12] W.B.R. Lickolish: A relationship between link polynomials, Math. Proc. Cambridge Philos. Soc. 100 (1986), 109-112.

[13] J.H. Przytycki and P. Traczyk: Invariants of links of Conway type, to appear in Kobe J. Math.

Department of Mathematics Osaka University

Toyonaka, Osaka 560, Japan 